\title{
Validation of Wendelstein 7-X fabrication and assembly stages by magnetic field calculations
}

\author{
T. Andreeva ${ }^{1}$, J. Kisslinger ${ }^{2}$ \\ ${ }^{1}$ Max-Planck-Institut für Plasmaphysik, Teilinstitut Greifswald, EURATOM Association \\ Wendelsteinstraße 1, D-17491 Greifswald \\ ${ }^{2}$ Max-Planck-Institut für Plasmaphysik, EURATOM Association \\ Boltzmannstraße 2, D-85748 Garching bei München
}

\section{Introduction.}

The Wendelstein 7-X stellarator, which is currently under construction in Greifswald, is a 5-period machine, and many of the planned operational plasma scenarios are characterized by a rotational transform $l / 2 p=1$ at the plasma boundary. Such magnetic configurations are very sensitive to the symmetry breaking perturbations caused by fabrication and assembly errors, which can occur at different stages of the device construction. As a consequence, new islands at any periodicity can be produced, existing islands can be modified, stochastic regions can be enhanced and power load onto the divertor plates can be increased. Therefore the high precision of the machine construction is a very important issue, and evaluation of the magnetic field is necessary for the continuous validation of the fabrication and assembly stages with respect to their impact on the magnetic field perturbation.

Analysis of the first fabricated winding packs (WPs) has shown that the fabrication errors can be divided into the systematical and statistical parts [1]. The systematic deviations add only negligible field components and don't perturb 5-fold symmetry of the machine, whilst the statistical deviations cause the disturbance of the machine periodicity.

For that estimation of the magnetic field perturbation the numerical procedure has been developed [2], which describes statistically the randomly distributed errors, taken within the given tolerances or uses the actual measurements available as an input parameter. Since the construction of the magnet system of $\mathrm{W} 7-\mathrm{X}$ is subdivided into two main phases - fabrication of components by industrial contractors and assembly of these components into the magnet system at the Greifswald site, the analysis of the magnetic field perturbation starts from the consideration of the impact of the WPs geometry deviations during the manufacturing stage.

\section{Impact of fabrication errors on the magnetic field perturbations.}

The actual geometry of currents path in each WP is simulated from the measurements of 8 surface points in 96 cross-sections, which were used as an input parameter for the mentioned above numerical procedure. Average absolute deviations of the manufactured non-planar and planar WPs from the designed (blue columns) and calculated average shape for each coil type (red columns) are shown at figure 1. 

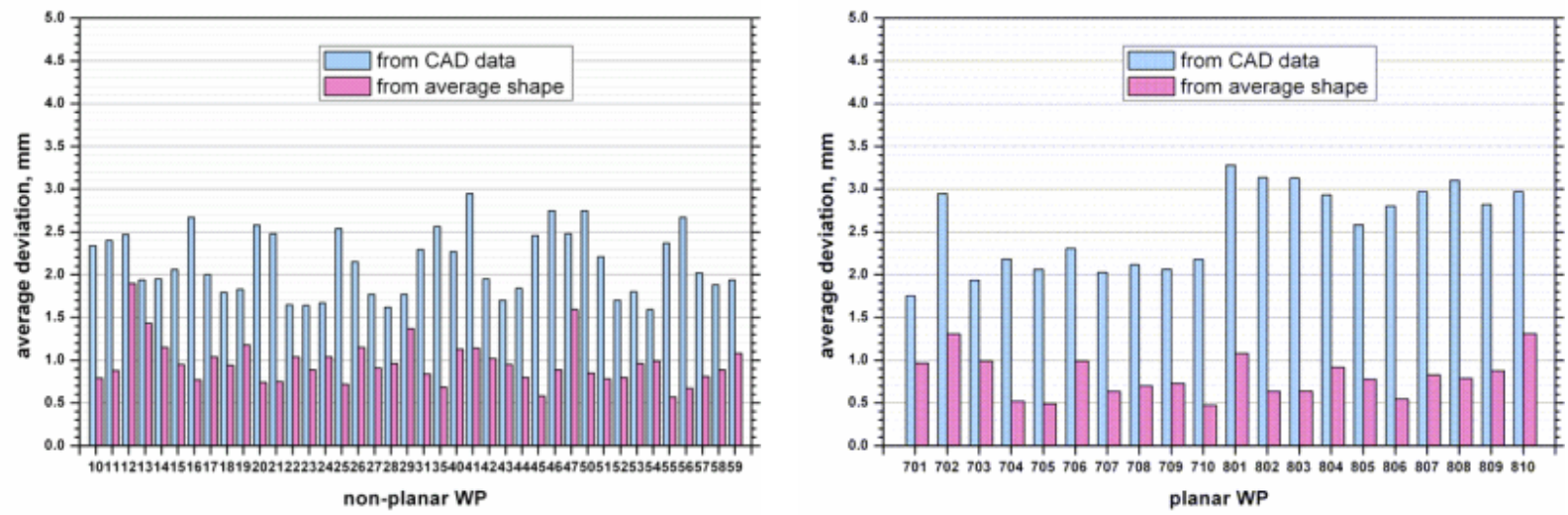

Fig.1 Absolute average deviations of the manufactured shape of the non-planar and planar winding packs.

Current status of 40 already fabricated non-planar WPs is that their absolute average deviations from the designed (CAD) coil shape are less than $3 \mathrm{~mm}$, and from the average shape found for each coil type - less than $2 \mathrm{~mm}$. For the planar WPs these numbers are 3.5 and 1.5 respectively, which is related on one side to the larger manufacturing tolerances during the production and on the other side - to the simpler geometrical shape. That is why the extracted statistical part (red columns) is smaller in comparison with the non-planar WPs.

The level of the perturbed magnetic fields as a function of the number of non-planar winding packs (WPs) manufactured is presented for two cases (fig. 2). In the first case we assume the not yet manufactured WPs to have exactly the designed geometry. In the second one they have an average shape which represents the systematic deviations. The investigation shows that the current level of the magnetic field perturbation corresponding to the 40 already manufactured WPs is around $0,8 \cdot 10^{-4}$ and can be expected at the end of the production stage of order $1 \cdot 10^{-4}$.
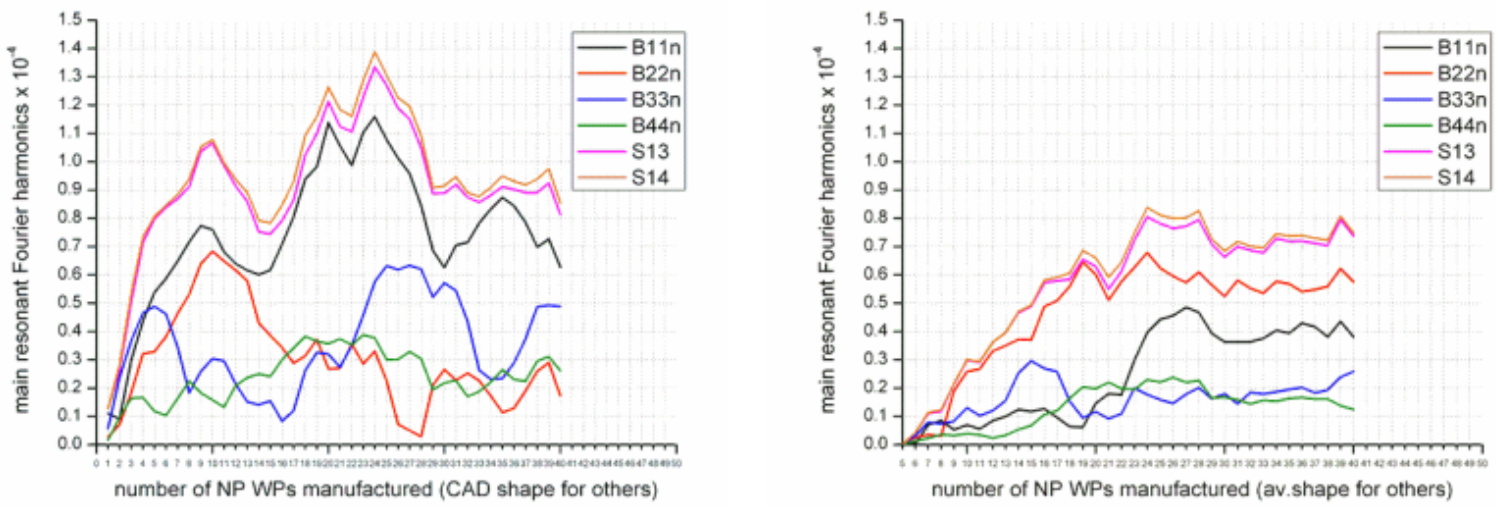

Fig.2 Magnetic field perturbation as a function of the number of non-planar WPs manufactured in case, when the rest, not-yet produced WPs, have the CAD shape (left graph) and in case, when the rest, not-yet produced WPs, have the average shape for each coil type (right graph). 
The current path calculated from the measurements at the fabrication stage will be the basis for all further estimations of the influence of the actual assembly procedure on the magnetic field perturbation.

The uncertainties related to the fabrication stage are the incompleteness of the measurements in some (few) points, measurement inaccuracy, variation of the ground insulation thickness, which for this calculations was assumed to be similar for all coils of the same type, and the deformation of the WP during the embedding process.

\section{Evaluation of the magnetic field during the assembly.}

Positioning errors impact in the magnetic field perturbation during the machine assembly will be done on the basis of the reference points measurements, which are supposed to be provided after each significant assembly step. For preliminary assessments of the magnetic field perturbations such assembly steps were combined into three stages and then the measurements of the reference points at the coils and the support ring were simulated. It was assumed that reference points are deviating randomly from their designed values in a sphere of $1.5 \mathrm{~mm}$, where any actual coordinate can be met with the same probability.

In addition there are the uncertainties in the determination of the reference point positions due to the finite device accuracy and readjustment of the measuring laser tracker system. These uncertainties were assumed respectively $0.5 \mathrm{~mm}, 1 \mathrm{~mm}$ and $0.8 \mathrm{~mm}$ for the first, second and the third simulated assembly stage, where the statistical values were taken also with the constant distribution function.

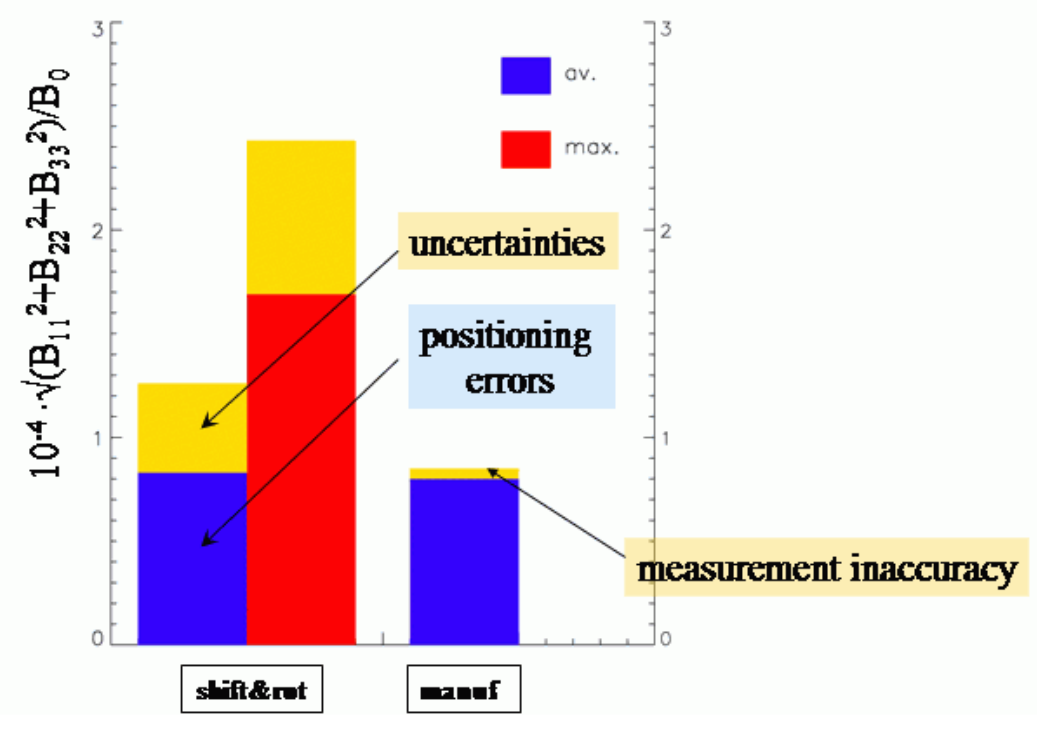

Fig.3 Simulation of the magnetic field perturbation due to the positioning errors.

Fig.3 demonstrates results of simulations for the cases when coils, half-modules and modules are shifted and rotated during the assembly. The vertical axis shows the magnetic 
field perturbation and the left blue and red columns correspond to the assembly positioning errors impact. The right blue column is presented just for the comparison of the positioning error impact with the manufacturing stage influence. Left blue column shows the average magnetic field perturbation due to the positioning errors and the yellow part above shows the average impact from the corresponding uncertainties. Red column represents the maximum perturbation found between 20 runs and the yellow part on the top - maximum value of the perturbed magnetic field due to the uncertainties, which reach more than $1 / 4$ of the value corresponding to the positioning error impact.

Another kind of assembly errors are the distortions due to the welding of the lateral supports. Each coils has two neighbouring coils to which it is connected by one or two welded lateral support elements. During the welding process the connection area will shrink and the influence of such a shrinkage is not balanced from both sides of the coil due to the different points of location of the lateral support connections. Therefore the coil shape or position can be changed during the welding process.

In order to simulate the magnetic field perturbation the following assumptions about the connections were done. Each coil of type 1 has two welded connections to the coil of type 1 of the next half-module, and 1 welded connection to the coil of type 2 in the same halfmodule. Coils of type 2, 3 and 4 have one welded connection to the adjacent coil on each side and coil of type 5 has only one welded connection to the coil of type 4 .

The influence of the welding process on the magnetic field perturbation has been investigated for two cases. Each coil has also two connections to the central support ring. In the first approach we assumed these connections to the central support ring to be rigid, hence shrinking during the welding leads only to the coil deformation. This deformation is maximum in points of location of the lateral support elements and " 0 " between the point of connection to the central support ring. The input parameter for the numerical procedure was the maximum shrinkage distance $\mathrm{a}_{\max }$ between two coils.

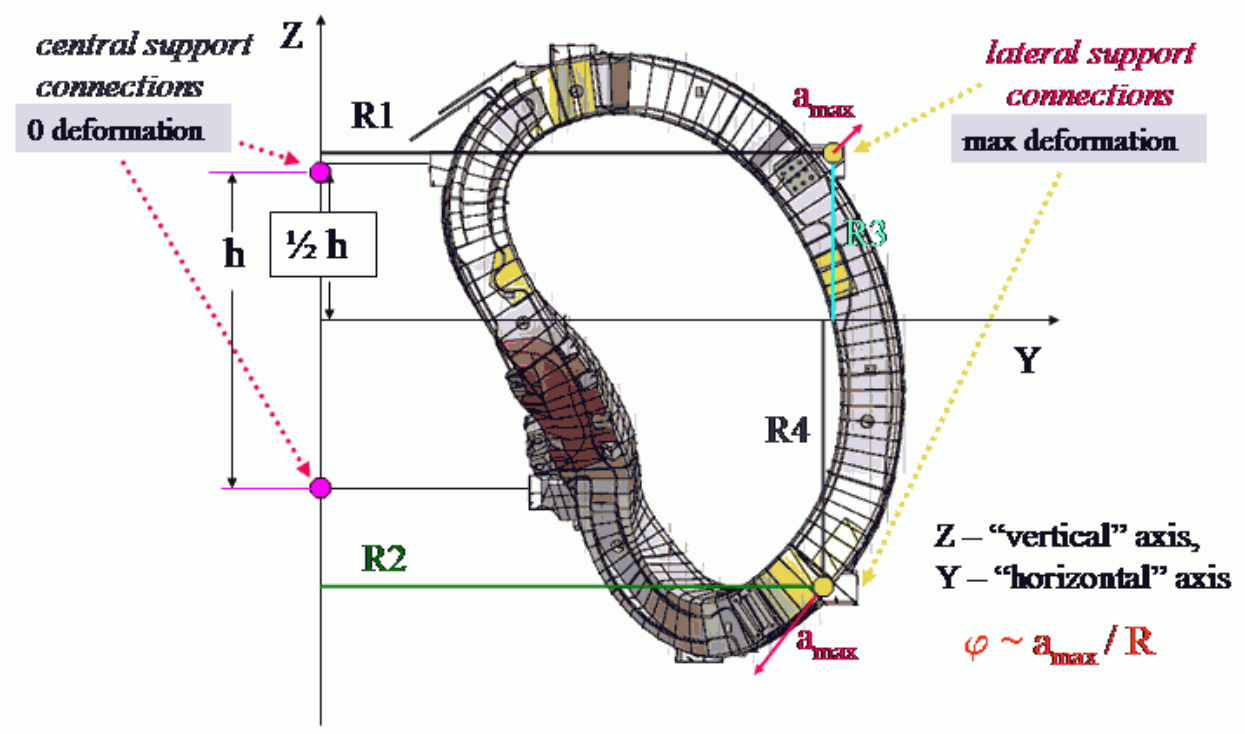

Fig.4 Scheme of the lateral supports welding modelling. 
In the second model we assumed the coils to be rigid, and connections to the central support ring to be deformed. Due to such a distortion coils can be rotated and hence their positions can be changed. The input parameter here was also the maximum shrinkage distance $a_{\max }$ in the lateral support elements.

Fig. 4 demonstrates the scheme of modelling. In case of coils rotation, angle of rotation is defined as $\varphi=a_{\max } / R$, where $R$ is a shortest distance to the axis of rotation. That is why two choices of rotation axis are considered: "vertical" - connecting the central support elements and "horizontal", which goes through the middle point of the distance between central support connections and middle point of the projection of the lateral support connection on the plane perpendicular to the "vertical" axis.

Main results of simulation are shown in Fig. 5. The magnetic field perturbation due to the welding of the lateral supports is shown for several input parameters $a_{\max }$ plotted on the horizontal axis. Influence of the coils deformation is shown by the blue colour. Green and black lines correspond to the coil rotation around vertical and horizontal axes. Shown value of the magnetic field perturbation is an average found between 20 runs. Dependently on model of distortion, $1 \mathrm{~mm}$ shrinkage distance leads to the magnetic field perturbation from 0.1 till $0.9 \cdot 10^{-4}$. In the actual procedure a combination of the coil deformation and rotation can happen, so the first approach is possibly an underestimation of the welding impact and value of $0.9 \cdot 10^{-4}$, corresponding to the pure rotation around horizontal axis, is an overestimation of the welding consequences.

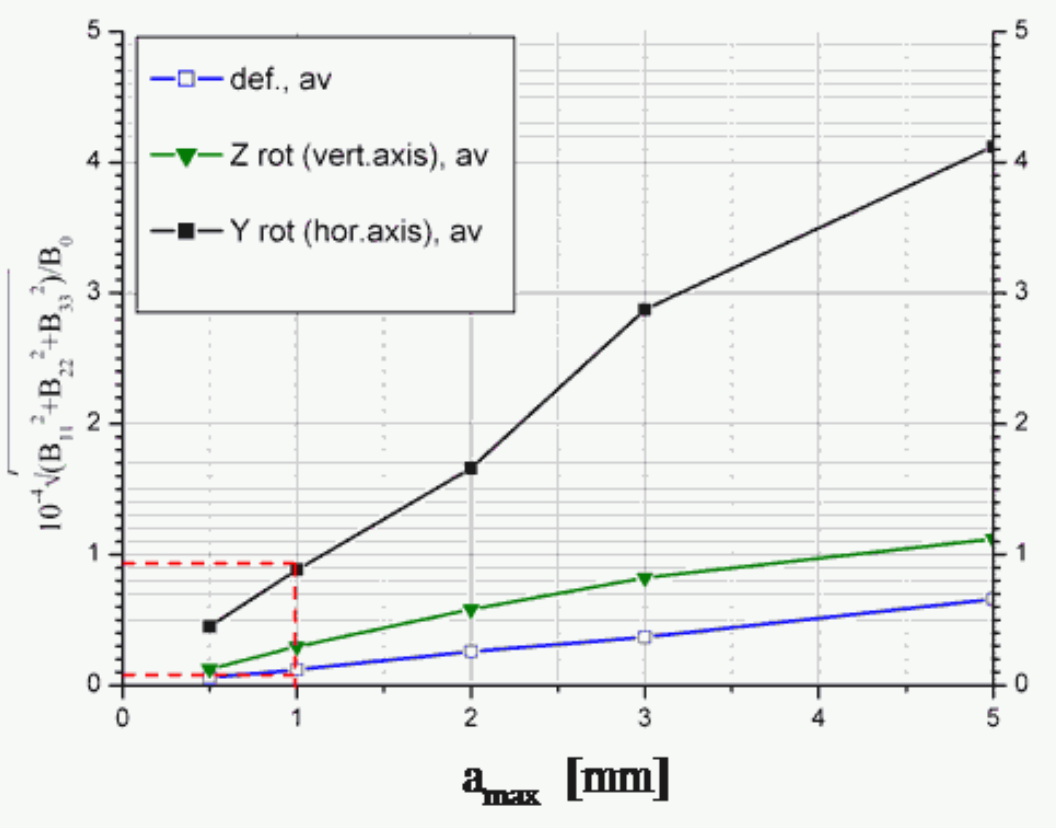

Fig.5 Magnetic field perturbation due to the welding of lateral supports. 


\section{Conclusions.}

Analysis of the fabrication errors of 40 from 50 non-planar and all 20 planar winding packs manufactured by present time has confirmed the existence of the systematical part in the manufacturing deviations. The level of the magnetic field perturbation due to the statistical part in manufacturing errors is expected of order $1 \cdot 10^{-4}$ by the end of the winding pack production stage. The current path calculated from the measurements of the winding pack actual geometry will be the basis for the evaluation of the magnetic field perturbation during the assembly procedure. Validation of different assembly steps will be done on the basis of the reference points measurements, which have to be provided after each action leading to distortion of the current path. Preliminary assessments of the possible positioning errors, lateral support welding and some uncertainties impact in the predictions of the final magnetic configuration show the necessity of well developed compensation procedure in order to minimise the magnetic field perturbation.

\section{References:}

1. Andreeva T., T. Bräuer, M. Endler, J. Kisslinger, Yu. Igitkhanov "Analysis of the magnetic field perturbations during the assembly of Wendelstein 7-X”, Fusion Science and Technology 46, 388-394 (2004).

2. Andreeva T., T. Bräuer and J. Kisslinger "Modelling of Magnetic Field Perturbations and Correction Possibilities in Wendelstein 7-X", 31-st EPS Conference on Plasma Physics and Controlled Fusion, London 2004, (Eds.) P. Norreys, H. Hutchinson. ECA 28G. European Physical Society, Geneva 2004, P-1.203. 